\title{
ENSAYOS DE PROCEDENCIAS DE Eucalyptus camaldulensis Dehnh EN LA ZONA SEMIARIDA DE CHILE
}

\author{
Santiago Barros A* \\ Patricio Rojas V.
}

\section{RESUMEN}

En 1984 se instalaron cuatro ensayos de procedencias de Eucalyptus camaldulensis Dehnh en la zona central de Chile (320 a $34^{\circ}$ lat S), donde la precipitación media anual varía entre 300 y $700 \mathrm{~mm}$.

La semilla, obtenida de CSIRO-Australia, correspondía a 14 procedencias, entre las cuales se incluían las más sobresalientes del clima tropical, Katherine, N.T. y Petford, QLD, del clima mediterráneo, Lake Albacutya, Vic., y del clima árido, Nueva Gales del Sur.

A los dos años, en las cuatro localidades ensayadas, la procedencia de mayor plasticidad en términos de supervivencia, crecimiento, forma y productividad fue Lake Albacutya, Vic (10666). En el mejor sitio su altura promedio a esa misma edad fue de $2,13 \mathrm{~m}$ con un crecimiento medio anual en altura de 1,12 m.

Las procedencias de menor crecimiento en todos los lugares de ensayo fueron: Agnew, W.A. (9856); NW Dodnadatta, S.A. (12828); N. de Quilpio, QLD (13264 y Fitzroy River Crossiana, W.A. (13250).

\section{ABSTRACT}

In 1984 four provenance trials of Eucalyptus camaldulensis Dehnh were established in central Chile ( $32^{\circ}$ to $340 \mathrm{~S}$. Lat.), where the mean annual rainfall varies from 300 to $700 \mathrm{~mm}$.

The seed for the trials was obtained from CSIRO. Fourteen provenances were studied. Some of them have often proved to be among the best provenances for their preferred climates. Tropical: Katherina, N.T. and Petford, QLD.; mediterranean: Lake Albacutya, Vic.; and arid: New South Wales.

At age two the best provenance in the four trials, was Lake Albacutya, Vic. (10666). It was the best in terms of form, survival and growth rate. On the best site those plants reached a height of $2.13 \mathrm{~m}$., with a yearly growing rate growing of $1.12 \mathrm{~m}$.

The slowest growing provenances in the four trials were Agnew, W.A. (9856); N. W. Dondnadatta, S.A. (12828); N. of Quilpio, QLD. (13264) and Fitzroy River Crossiana, W.A. (13250).

* División Silvicultura. Institu to Forestal, Huérfanos 554, Santiago - Chile, Tel. 396189. 


\section{INTRODUCCION}

Dentro de la red de ensayos de introducción de especies establecida por el Instituto Forestal desde el año 1962, se ha probado un considerable número de diferentes especies forestales en distintas zonas del país. En la zona semiárida se han ensayado principalmente especies del género Eucalyptus y los resultados obtenidos hasta ahora indican que Eucalyptus camaldulensis, Eucalyptus cladocalyx y Eucalyptus sideroxylon son las especies forestales exóticas de mayor interés para esta zona, principalmente para los sectores de secano interior con precipitaciones inferiores a $500 \mathrm{~mm}$ anuales (INFOR, 1986).

Sin embargo, el escaso conocimiento que se tiene en el país acerca de la silvicultura de estas especies no justifica su inclusión en programas de forestación a gran escala, razón por la cual se está trabajando en el desarrollo de técnicas intensivas de establecimientos de plantaciones y en la selección de procedencias de semillas adecuadas para las condiciones de sitio propias de esta zona. En el año 1984 se establecieron ensayos de procedencias de Eucalyptus camaldulensis en cuatro lugares de la zona semiárida del país, cuyos resultados a los dos años de edad se analizan en el presente trabajo.

El Eucalyptus camaldulensis Dehnh. ("River Red Gum"), junto con ser la especie del género Eucalyptus más difundida en el mundo (NAS, 1980), es una de las especies de eucalipto de más amplia distribución natural en Australia, donde se la encuentra en todos los estados con la excepción de Tasmania. Ocupa un rango latitudinal entre los $12030^{\prime}$ y los 380 de Latitud Sur y ocurre naturalmente en altitudes de 20 a 700 m.s.n.m. Esta especie puede prosperar bien bajo una variedad de condiciones climáticas, desde las tropicales a las subtropicales, con temperaturas máximas medias de verano de 27 a $40^{\circ} \mathrm{C}$ y mínimas medias invernales de 3 a $15^{\circ} \mathrm{C}$ y con precipitaciones medias anuales de 150 a $1250 \mathrm{~mm}$, de régimen invernal en la parte sur y estival en los estados del norte.

La especie ha sido ampliamente utilizada en plantaciones fuera de Australia, principalmente en países de la cuenca del Mediterráneo. Actualmente existen unas 500 mil hectáreas plantadas en el mundo (Nawaz, 1963). Las repoblaciones más extensas se encuentran en España (114.000 ha) y Marruecos $(87.000 \mathrm{ha})$, donde se emplea para la producción de pulpa, al igual que en Portugal, a pesar de que su madera es más dura, pesada y coloreada que la de $E$. globulus (NAS, 1980). Otros países que han incluido esta especie en sus programas de forestación son Argentina, Israel y México, donde se utiliza en la fabricación de tableros (Turnbull and Pryor, 1978). También debido al alto poder calorífico de la madera $(4.800 \mathrm{Kcal} / \mathrm{kg})$, la especie es usada con fines energéticos.

El interés por plantar Eucalyptus camaldulensis en diferentes partes del mundo radica en su rápido crecimiento en sitios pobres y de escasa precipitación, donde los periodos secos son prolongados; en su capacidad de retoñar adecuadamente después de la explotación; en la utilidad de su madera para una variedad de usos y en la tolerancia que presenta la especie a inundaciones periódicas y a cierta salinidad en el suelo (Turnbull and Pryor, 1978).

Dependiendo de la procedencia de la semilla y del sitio en que se le plante, $E$. camaldulensis puede crecer muy rápido. Durante los primeros diez años pueden mantenerse incrementos anuales de $2 \mathrm{~m}$ en altura y de $2 \mathrm{~cm}$ en diámetro. Rendimientos volumétricos de hasta $30 \mathrm{~m}^{3} / \mathrm{ha} /$ año han sido medidos en buenos sitios (FAO, 1979). En sitios pobres y secos, los incrementos son mucho menores. En un ensayo de 13 procedencias efectuado en Peshawar (Pakistán), el rango del incremento medio anual fue de 5.5 y $15.5 \mathrm{~m}^{3} /$ ha/año (Siddiqui K.M. et al., 1979).

La amplitud de la distribución natural de esta especie en su país de origen otorga una especial importancia a la selección adecuada de procedencias de semilla para cada zona que se intente repoblar, ya que éste es un factor que puede originar significativas diferencias de adaptación y de productividad en una determinada zona, las cuales se ven reflejadas en el crecimiento, 
morfología y calidad de los árboles, en su tolerancia al medio (frío, sequia, plagas u otros factores) y en la calidad de la madera.

En esta perspectiva, el objetivo de este trabajo fue evaluar el rendimiento y desarrollo de diferentes procedencias de Eucalyptus camaldulensis, con el fin de seleccionar las más adecuadas para la forestación en distintos sectores de la zona semiárida del país y entregar sugerencias para nuevas investigaciones de acuerdo con los resultados obtenidos.

\section{MATERIAL Y METODO}

Según Burley y Wood (1979), las etapas que deben cumplirse para la selección de la procedencia más adecuada de una especie son las siguientes:

a) Fase de Alcance Completo

Su objetivo es determinar la amplitud y el patrón de variabilidad genética entre procedencias (poblaciones) de la especie en su distribución natural.

El número de procedencias que se deben ensayar varía de 10 a 30 y la duración de la fase para obtener algunas conclusiones varía entre 0,25 y 0,5 veces la rotación estimada de la especie.

b) Fase de Alcance Restringido

Su objeto es determinar sub-regiones y finalmente aquellas procedencias más adecuadas para el área de estudio.

El número de procedencias que se deben ensayar varía entre 3 y 5 y la duración de esta fase es de 0,5 veces la rotación estimada de la especie.

c) Fase de Comprobación

Su objeto es confirmar, bajo las condiciones normales de plantación, los resultados obte. nidos por la procedencia seleccionada.

El número de procedencias que se deben ensayar es $1 \mathrm{y}$ en algunos casos 2 .

Generalmente las experiencias que se desarrollan en esta fase son lạs siguientes:

- Ensayos de viverización

- Métodos de preparación del suelo

- Métodos de plantación

- Ensayos de espaciamientos y raleos

- Ensayos de control de competencia

- Ensayos de poda

- Ensayos de fertilizantes y herbicidas

Después de la fase de comprobación, se efectúan plantaciones piloto como paso intermedio entre la fase experimental y las plantaciones a escala industrial.

Estas plantaciones pueden demostrar a los potenciales inversionistas la factibilidad de un proyecto a gran escala y también proporcionan la oportunidad de desarrollar sistemas de manejo y técnicas silviculturales.

Ubicación y descripción de los ensayos: Los ensayos se establecieron en cuatro lugares de la zona semiárida de Chile, entre los 32 y $34^{\circ}$ de latitud (Mapa ubicación).

La zona de estudio corresponde al tipo bioclimático Mediterráneo (Di Castri, 1975), que se caracteriza por una baja precipitación anual $(300 \cdot 7000 \mathrm{~mm})$ distribuida fundamentalmente en los meses de invierno, por lo que existe un largo período seco de 5 a 8 meses.

Los suelos de la zona están severamente degradados por sobrepastoreo, presentando un horizonte A de 20 a $40 \mathrm{~cm}$ de profundidad, neutro a ligeramente ácido y de muy bajo contenido de materia orgánica. El horizonte B normalmente presenta una reacción mas alcalina. En una estructura más fuerte y texturas más gruesas. 
La vegetación natural de la zona, fuertemente degradada debido a la sobreutilización, está representada por una formación mesomórfica en la que las especies de mayor importancia son Acacia caven (Espino), Quillaja saponaria (Quillay) y Peumus boldus (Boldo).

En la parte norte predomina la primera de las especies nombradas, constituyendo el tipo forestal denominado Estepa de Acacia caven. En la parte central y sur de la zona estas especies se combinan constituyendo el tipo Bosque Esclorófilo Mixto.

En el Cuadro No 1, Figura № 1 siguientes, se caracterizan los lugares de ensayo desde el punto de vista fisiográfico, edáfico y climático.

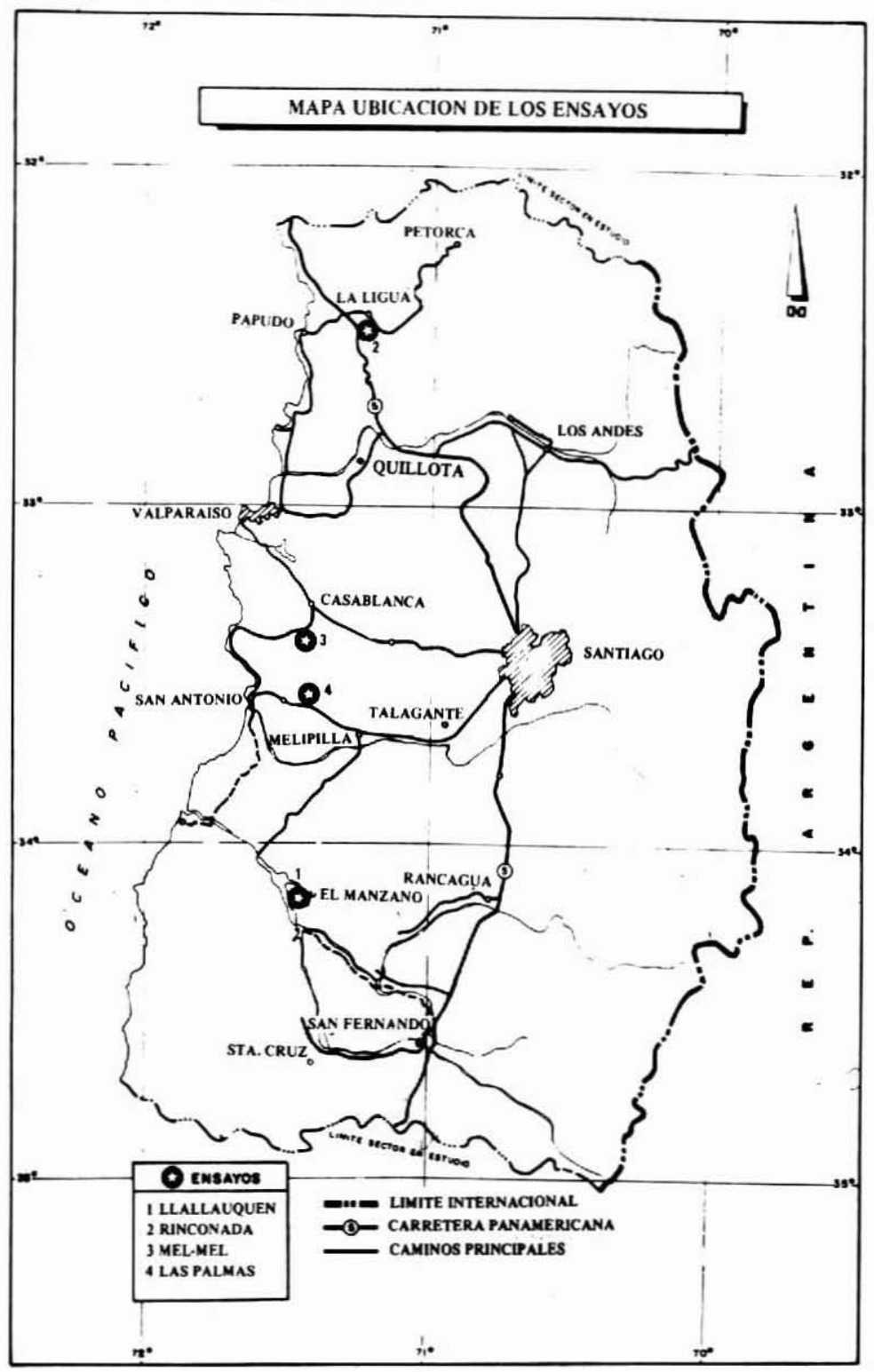




\section{CUADRO 1 \\ UBICACION Y CARACTERIZACION DE LOS ENSAYOS}

\begin{tabular}{|c|c|c|c|c|c|}
\hline & & $\begin{array}{l}\text { Enaryo ! } \\
\text { Uallavquén }\end{array}$ & $\begin{array}{c}\text { Enenyo } 2 \\
\text { Rinconada }\end{array}$ & $\begin{array}{l}\text { Ensayo } 3 \\
\text { Mel-Mel }\end{array}$ & $\begin{array}{l}\text { Enacyo } 4 \\
\text { Lea Palmas }\end{array}$ \\
\hline I. & $\begin{array}{l}\text { Ubicación } \\
\text { Latitud Sur } \\
\text { Longitud Oeste }\end{array}$ & $\begin{array}{ll}34^{\circ} & 15^{\circ} \\
71^{\circ} & 21^{\circ}\end{array}$ & $\begin{array}{ll}32^{\circ} & 30^{\prime} \\
71^{\circ} & 15\end{array}$ & $\begin{array}{ll}33^{\circ} & 22^{\circ} \\
71^{\circ} & 23^{\circ}\end{array}$ & $\begin{array}{ll}33^{\circ} & 36^{\prime} \\
71^{\circ} & 22^{\prime}\end{array}$ \\
\hline 2. & $\begin{array}{l}\text { Fisiografia } \\
\text { Pendiente }(\% / 0) \\
\text { Exposicion } \\
\text { Relieve }\end{array}$ & $\begin{array}{l}5 \cdot 10 \\
\text { Noreste } \\
\text { Plano }\end{array}$ & $\begin{array}{c}\text { Media } 26 \text { Míx. } 35 \\
\text { Sur } \\
\text { Lomaje }\end{array}$ & $\begin{array}{l}20-30 \\
\text { Noreste } \\
\text { Lomaje }\end{array}$ & $\begin{array}{c}0-5 \\
- \\
\text { Plano }\end{array}$ \\
\hline 3. & $\begin{array}{l}\text { Suelos } \\
\text { Gran grupo } \\
\text { Características del } \\
\text { Horizonte ? }\end{array}$ & $\begin{array}{l}\text { Pardo no cálcico } \\
\text { Profundidad del auelo de } \\
20-40 \mathrm{~cm} \text {; textura mode- } \\
\text { radamente grues variando } \\
\text { de franco arenos fina a } \\
\text { gruesa: eatructura de blo- } \\
\text { ques angulares y subangula- } \\
\text { res: pH } 5,4.6,0 \text {; color } \\
\text { pardo oscuro a pardo ama- } \\
\text { rillento presenta limitacio- } \\
\text { nes por etosión y/o topo- } \\
\text { erofia(1). }\end{array}$ & 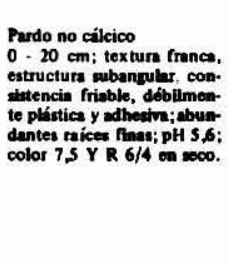 & 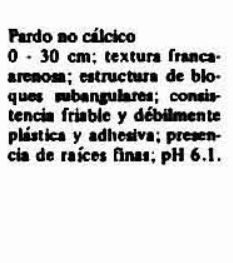 & $\begin{array}{l}\text { Pardo no cálcico } \\
0 \text { - } 30 \mathrm{~cm} \text {, textura franca- } \\
\text { arenosa; estructura de blo- } \\
\text { ques angulares y aubangula- } \\
\text { res; consiatencia friable, dó- } \\
\text { bilmeante plástica y adhesi- } \\
\text { va; raices abundantes, pH } \\
6,4 \text {; color } 5 \text { Y } 4 / 4 \text { en } \\
\text { húmedo. }\end{array}$ \\
\hline & $\begin{array}{l}\text { Caracteristicas del } \\
\text { Horizonte } 3\end{array}$ & & $\begin{array}{l}20-60 \mathrm{~cm} \text {; textura arcillo- } \\
\text { sa, eatructura priamática: } \\
\text { consiatencia firme. fuerte, } \\
\text { plástica y adhesivo: raices } \\
\text { escases pH S,7, colos S Y R } \\
4 / 4 \text { en weco. } \\
60 \text { y más cin.. formado por } \\
\text { maicillo muy descompues- } \\
\text { to, con presencia de piedra } \\
\text { y no hay presencia de raí- } \\
\text { ces (2). }\end{array}$ & $\begin{array}{l}30.70 \mathrm{~cm} \text {; formada por } \\
\text { maicillo grueso con abun- } \\
\text { dante cuarzo y una peque- } \\
\text { la matriz de material más } \\
\text { fino; no hay presencia de } \\
\text { raíces. pH } 6,1 \text {. (2). }\end{array}$ & $\begin{array}{l}30 \text {. } 75 \mathrm{~cm} \text {; textura franco- } \\
\text { arcillo-arenosa; estructura } \\
\text { de bloques angulares y wub- } \\
\text { angulares consiatencia fria- } \\
\text { ble, fuerte, plástico y adhe- } \\
\text { sivo: presencia de raices } \\
\text { finas, pH } 6,5 \text {; color } 2.5 \mathrm{Y} \\
\mathrm{R} 5 / 6 \text { en húmedo. } \\
75-130 \mathrm{~cm} \text {, predomina un } \\
\text { maicillo con abundante } \\
\text { cuarzo y muy denso. no } \\
\text { hay presencia de raices (2). }\end{array}$ \\
\hline 4. & $\begin{array}{l}\text { Clima } \\
\text { Tipo Bloclimático } \\
\text { Precipitación media anual }(\mathrm{mm}) \\
\text { Temperatura media anual }\left({ }^{\circ} \mathrm{C}\right) \\
\text { Meses secos }\end{array}$ & $\begin{array}{c}\text { Mediterrinco subhúmedo } \\
513,2 \\
150 \\
6.7(3)\end{array}$ & $\begin{array}{c}\text { Mediterráneo semiárido } \\
\begin{array}{c}328,8 \\
15,1^{0} \\
7.8(4)\end{array}\end{array}$ & $\begin{array}{c}\text { Mediterrineo semiárido } \\
729,5 \\
13,4^{\circ} \\
5.6(5)\end{array}$ & $\begin{array}{l}\text { Mediterráneo subhúmedo } \\
364,8 \\
14,70 \\
6.7 \text { (5) }\end{array}$ \\
\hline
\end{tabular}

IUENTE: (I) CIREN-CORFO 1980

$\begin{array}{ll}\text { (2) Jorge Toro } & 1986 \\ \text { (3) Endesa } & 1986 \\ \text { (4) CIREN-CORFO } & 1979 \\ \text { (S) Direccion Gral. } & \begin{array}{l}\text { de Nguas } \\ \text { de }\end{array} \\ \end{array}$

FIGURA 1

\section{DIAGRAMAS OMBROTERMICOS REPRESENTATIVOS DE LOS LUGARES DE ENSAYOS}

I. LLALAUQUEN

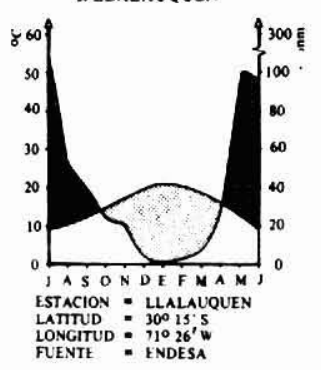

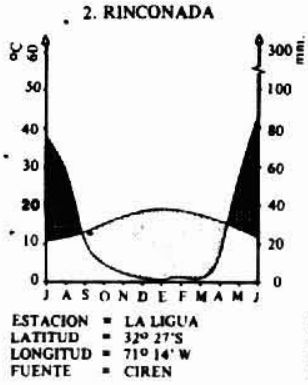

3. MEL-MEL

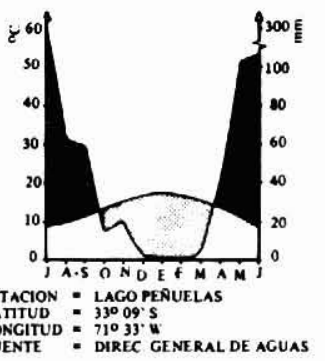

4. LAS PALMAS

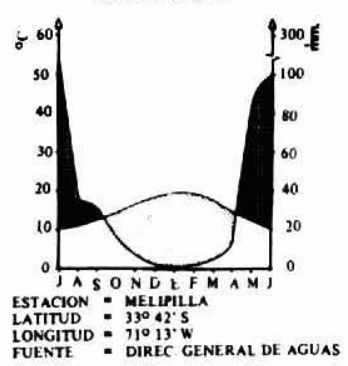


Procedencias Ensayadas: En los cuatro lugares se ensayó una colección de 14 procedencias de semillas obtenidas de CSIRO - Australia. Sin embargo, debido a que las muestras de semillas fueron limitadas, atgunas de las procedencias no están replicadas en todos los ensayos.

\section{CUADRO 2 \\ PROCEDENCIAS Eucalyptus camaldulensis}

\begin{tabular}{|c|c|c|c|c|c|c|c|c|c|c|}
\hline Localidad y Estado & $\left|\begin{array}{l}\text { Latitud } \\
\left.0^{\circ} \cdot S\right)\end{array}\right|$ & $\begin{array}{c}\text { Longitud } \\
(0 .\end{array}$ & $\begin{array}{l}\text { Altitud } \\
\text { (msnm) }\end{array}$ & $\begin{array}{c}\text { Precipitación } \\
\text { (mm/aho) }\end{array}$ & \begin{tabular}{|} 
Temper \\
Mixima media \\
mes más cilido \\
$\left({ }^{\circ} \mathrm{C}\right)$
\end{tabular} & $\begin{array}{l}\text { raturas } \\
\text { Minima media } \\
\text { mes mís frio } \\
\left({ }^{\circ} \mathrm{C}\right)\end{array}$ & Ualauquén & $\begin{array}{r}\text { Luger de En } \\
\text { La Rinconada }\end{array}$ & $\begin{array}{l}\text { nsayo } \\
\text { Mel-Mel }\end{array}$ & Las Palmas \\
\hline $\begin{array}{l}\text { Darling River Bourke, NSW } \\
\text { Minindee, NSW } \\
\text { Lake Albacutya, VIC. } \\
\text { NW Dodnadatta, SA } \\
\text { Agnew, WA } \\
\text { Lake Albacutya, VIC } \\
\text { Port Lincoln, SA } \\
\text { Fitzroy River Crossina, WA } \\
\text { EmuCreek Petford, QLD } \\
\text { Wof Wiluma, WA } \\
\text { Nof Quilpio, QLD } \\
\text { Umberumberka Creek, NSW } \\
\text { wiluma, WA } \\
\text { Sof Katherine, NT }\end{array}$ & $\begin{array}{ll}30 & 50 \\
32 & 25 \\
35 & 54 \\
26 & 57 \\
28 & 30 \\
35 & 44 \\
34 & 35 \\
18 & 11 \\
17 & 20 \\
26 & 33 \\
25 & 58 \\
31 & 57 \\
26 & 00 \\
14 & 30\end{array}$ & $\begin{array}{ll}145 & 57 \\
145 & 30 \\
142 & 00 \\
133 & 32 \\
120 & 57 \\
142 & 20 \\
135 & 38 \\
125 & 36 \\
144 & 68 \\
120 & 30 \\
144 & 35 \\
141 & 28 \\
120 & 00 \\
132 & 15\end{array}$ & $\begin{array}{r}110 \\
60 \\
70 \\
300 \\
490 \\
70 \\
90 \\
150 \\
460 \\
550 \\
260 \\
304 \\
- \\
110\end{array}$ & $\begin{array}{r}349 \\
237 \\
332 \\
129 \\
228 \\
332 \\
484 \\
519 \\
1427 \\
239 \\
291 \\
237 \\
239 \\
352\end{array}$ & $\begin{array}{l}36,8 \\
32,9 \\
32,4 \\
38,0 \\
38,3 \\
32,4 \\
24,9 \\
40,9 \\
29,6 \\
37,2 \\
37,9 \\
32,9 \\
37,2 \\
38,1\end{array}$ & $\begin{array}{r}4,5 \\
5,1 \\
4,2 \\
6,0 \\
6,6 \\
4,2 \\
8,4 \\
81.1 \\
11.1 \\
10,4 \\
4,9 \\
6,3 \\
5,1 \\
4,9 \\
13,2\end{array}$ & $\begin{array}{l}x \\
x \\
x \\
x \\
x \\
x \\
x \\
x\end{array}$ & $\begin{array}{c}\cdot x \\
x \\
x \\
x \\
x \\
x \\
x \\
x\end{array}$ & $\begin{array}{l}\mathrm{x} \\
\mathrm{x} \\
\mathrm{x} \\
\mathrm{x} \\
\mathrm{x} \\
\mathrm{x} \\
\mathrm{x} \\
\mathrm{x} \\
\mathrm{x} \\
\mathrm{x}\end{array}$ & $\begin{array}{l}x \\
x \\
x \\
x \\
x \\
x \\
x\end{array}$ \\
\hline
\end{tabular}

Las semillas fueron viverizadas durante el período diciembre 1983-mayo 1984. Las plantas fueron producidas en bolsas de polietileno de $10 \times 20 \mathrm{~cm}$ usando un sustrato compuesto por un $60 \%$ de tierra común, $20 \%$ o.de arena y $20 \%$ de tierra vegetal.

La vegetación arbustiva y arbórea de los lugares de ensayos fue eliminada a través de un roce manual. La plantación se efectuó durante los meses de junio y julio de 1984, coincidiendo con el período de mayor pluviosidad.

Diseño Experimental: Los ensayos fueron establecidos con un diseño completamente aleatorizado con 3 repeticiones, aunque cuando existían diferencias notorias en el lugar, debidas a la pendiente, exposición u otros factores, se empleó un diseño de bloques al azar.

La unidad experimental fue de 20 plantas ( 2 hileras de 10 plantas) espaciadas a $3 \times 3 \mathrm{~m}$. El método de establecimiento de las plantaciones y el diseño experimental de cada ensayo se detallan en el Cuadro $\mathrm{N}^{\circ} 3$.

Variables Analizadas: Los ensayos se controlaron una vez terminada la primera temporada de crecimiento (control año 1985) y una vez terminada la segunda temporada de crecimiento (control año 1986). En el primer control se midieron las siguientes variables, en las tres repeticiones.

a) Supervivencia $(\%)$

b) Altura total de las plantas (m)

En el segundo control, además de las variables mencionadas, se agregaron:

c) Diámetro de cuello, medido con pie de metro $(\mathrm{cm})$

d) Incremento en altura entre el primer y segundo año (m)

e) Un índice de crecimiento, como estimador de la biomasa, calculado a partir del diámetro de cuello al cuadrado multiplicado por la altura total $\left(\mathrm{dc}^{2} \times \mathrm{H}\right)$.

Análisis Estadistico. Debido a las restricciones impuestas por la heterogeneidad de los ensayos, en términos del tamaño de la unidad experimental, diseño estadístico y método de establecimiento, se éfectuó un análisis comparativo de las procedencias dentro de cada sitio 


\section{CUADRO 1 \\ UBICACION Y CARACTERIZACION DE LOS ENSAYOS}

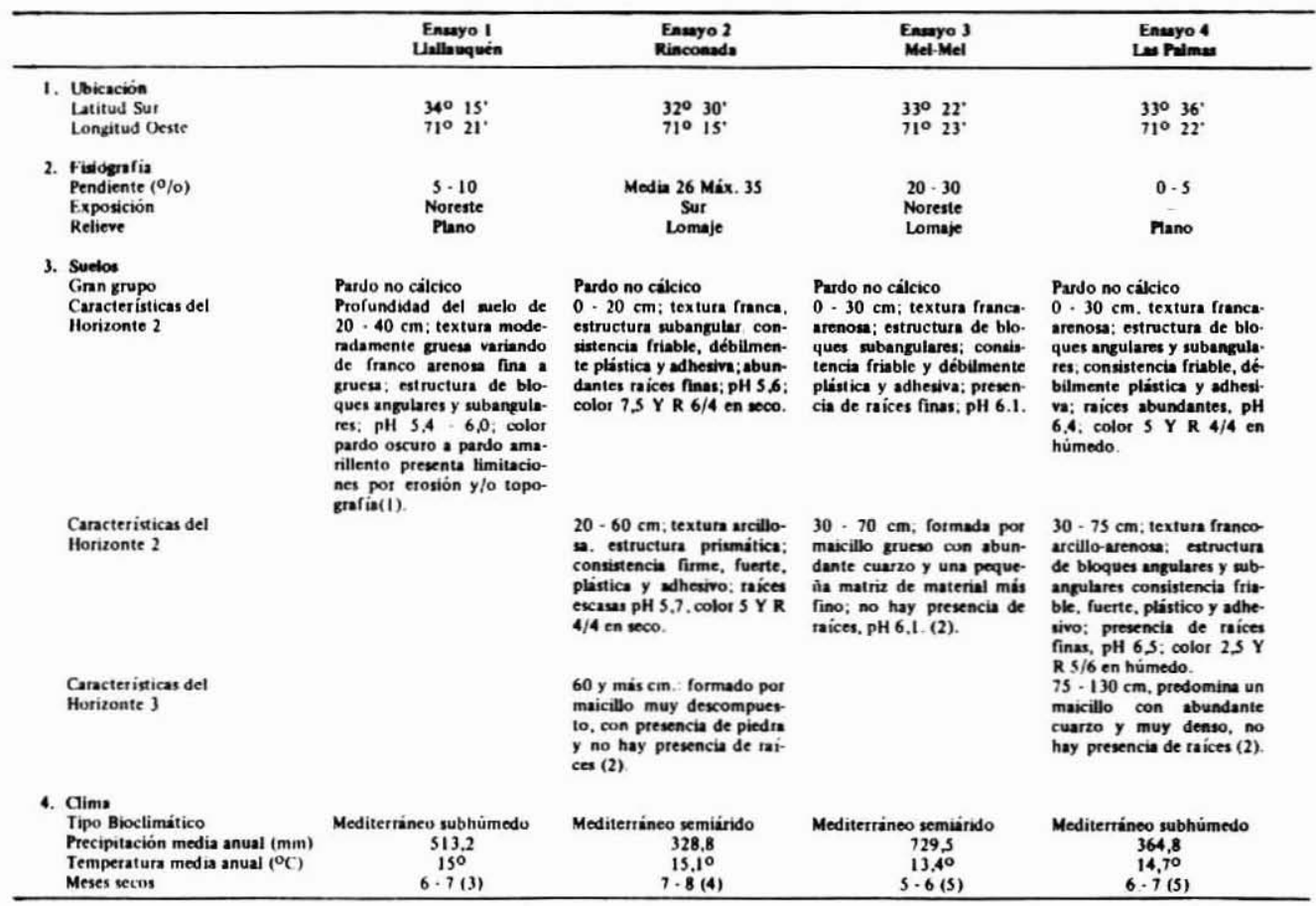

IUENTE: (1) CIREN-CORFO 1980

$\begin{array}{ll}\text { (2) Jorge Toro } & 1986 \\ \text { (3) Endesa } & 1986\end{array}$

$\begin{array}{ll}\text { (3) Endcsa - CORFO } & 1986 \\ \text { (4) CIREN-COR9 }\end{array}$

(S) Direccion Gral. de Aguas $\quad 1986$

FIGURA 1

\section{DIAGRAMAS OMBROTERMICOS REPRESENTATIVOS DE LOS LUGARES DE ENSAYOS}

I. LLALAUQUEN

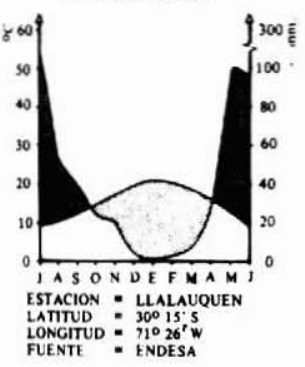

2. RINCONADA

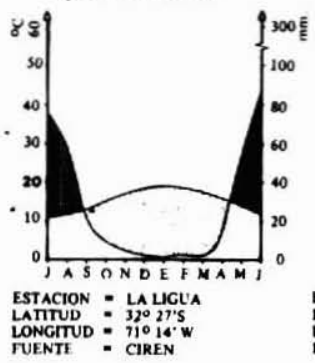

3. MEL-MEL

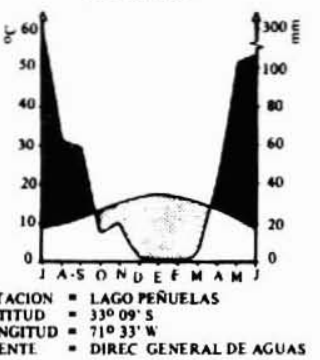

4. LAS PALMAS

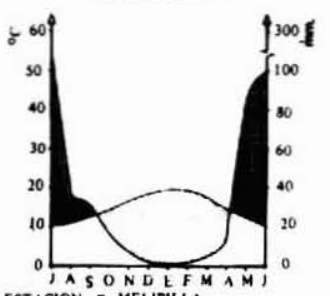

ESTACION - MELLPILLA

LATITUD = $33^{\circ} 42^{2}$.

FUENTE : DIREC. GENERAL DE AGUAS 
Procedencias Ensayadas: En los cuatro lugares se ensayó una colección de 14 procedencias de semillas obtenidas de CSIRO - Australia. Sin embargo, debido a que las muestras de semillas fueron limitadas, algunas de las procedencias no están replicadas en todos los ensayos.

\section{CUADRO 2 \\ PROCEDENCIAS Eucalyptus camaldulensis}

\begin{tabular}{|c|c|c|c|c|c|c|c|c|c|c|}
\hline Localidad y Estado & $\begin{array}{l}\text { Latitud } \\
(0 ; \mathrm{S})\end{array}$ & $\begin{array}{l}\text { Longitud } \\
(0 \cdot E)\end{array}$ & $\begin{array}{l}\text { Altitud } \\
\text { (msnm) }\end{array}$ & $\begin{array}{l}\text { Precipitación } \\
\text { (mm/año) }\end{array}$ & $\begin{array}{l}\text { Temper } \\
\text { Máxima media } \\
\text { mes mis calido } \\
\left.\text { ( }{ }^{\circ} \mathrm{C}\right)\end{array}$ & $\begin{array}{l}\text { Minima media } \\
\text { mes más frio } \\
\text { (O C) }\end{array}$ & Ualauquén & $\begin{array}{l}\text { Lugar de En } \\
\text { La Rinconada }\end{array}$ & $\begin{array}{l}\text { nsayo } \\
\text { Mel-Mel }\end{array}$ & Las Palmas \\
\hline $\begin{array}{l}\text { Darling River Bourke, NSW } \\
\text { Minindee, NSW } \\
\text { Lake Albacutya, VIC. } \\
\text { NW Dodnadatta, SA } \\
\text { Agnew, WA } \\
\text { Lake Albacutya, VIC } \\
\text { Port Lincoln, SA } \\
\text { Fitzroy River Crossina, WA } \\
\text { EmuCreek Petford, QLD } \\
\text { Wof Wiluma, WA } \\
\text { Nof Quilpio, QLD } \\
\text { Umberumberka Creek, NSW } \\
\text { Wiluma, WA } \\
\text { Sof Katherine, NT }\end{array}$ & $\begin{array}{ll}30 & 50 \\
32 & 25 \\
35 & 54 \\
26 & 57 \\
28 & 30 \\
35 & 44 \\
34 & 35 \\
18 & 11 \\
17 & 20 \\
26 & 33 \\
25 & 58 \\
31 & 57 \\
26 & 00 \\
14 & 30\end{array}$ & $\begin{array}{ll}145 & 57 \\
145 & 30 \\
142 & 00 \\
133 & 32 \\
120 & 57 \\
142 & 20 \\
135 & 38 \\
125 & 36 \\
144 & 68 \\
120 & 30 \\
144 & 35 \\
141 & 28 \\
120 & 00 \\
132 & 15\end{array}$ & $\begin{array}{r}110 \\
60 \\
70 \\
300 \\
490 \\
70 \\
90 \\
150 \\
460 \\
550 \\
260 \\
304 \\
110\end{array}$ & $\begin{array}{r}349 \\
237 \\
332 \\
129 \\
228 \\
332 \\
484 \\
519 \\
1427 \\
239 \\
291 \\
237 \\
239 \\
352\end{array}$ & $\begin{array}{l}36,8 \\
32,9 \\
32,4 \\
38,0 \\
38,3 \\
32,4 \\
24,9 \\
40,9 \\
29,6 \\
37,2 \\
37,9 \\
32,9 \\
37,2 \\
38,1\end{array}$ & $\begin{array}{r}4,5 \\
5,1 \\
4,2 \\
6,0 \\
6,6 \\
4,2 \\
8,4 \\
11,1 \\
10,4 \\
4,9 \\
6,3 \\
5,1 \\
4,9 \\
13,2\end{array}$ & $\begin{array}{l}\mathbf{x} \\
\mathbf{x} \\
\mathbf{x} \\
\mathbf{x} \\
\mathbf{x} \\
\mathbf{x} \\
\mathbf{x} \\
\mathbf{x}\end{array}$ & $\begin{array}{c}\cdot x \\
x \\
x \\
x \\
x \\
x \\
x\end{array}$ & $\begin{array}{l}x \\
x \\
x \\
x \\
x \\
x \\
x \\
x \\
x \\
x\end{array}$ & $\begin{array}{l}x \\
x \\
x \\
x \\
x \\
x \\
x \\
\\
x \\
x \\
x \\
x\end{array}$ \\
\hline
\end{tabular}

Las semillas fueron viverizadas durante el periodo diciembre 1983-mayo 1984. Las plantas fueron producidas en bolsas de polietileno de $10 \times 20 \mathrm{~cm}$ usando un sustrato compuesto por un 60\% de tierra común, $20 \%$ o.de arena y $20 \%$ de tierra vegetal.

La vegetación arbustiva y arbórea de los lugares de ensayos fue eliminada a través de un roce manual. La plantación se efectuó durante los meses de junio y julio de 1984, coincidiendo con el período de mayor pluviosidad.

Diseño Experimental: Los ensayos fueron establecidos con un diseño completamente aleatorizado con 3 repeticiones, aunque cuando existían diferencias notorias en el lugar, debidas a la pendiente, exposición u otros factores, se empleó un diseño de bloques al azar.

La unidad experimental fue de 20 plantas ( 2 hileras de 10 plantas) espaciadas a $3 \times 3 \mathrm{~m}$. El método de establecimiento de las plantaciones y el diseño experimental de cada ensayo se detallan en el Cuadro No 3.

Variables Analizadas: Los ensayos se controlaron una vez terminada la primera temporada de crecimiento (control año 1985) y una vez terminada la segunda temporada de crecimiento (control año 1986). En el primer control se midieron las siguientes variables, en las tres repeticiones.

a) Supervivencia $(\%)$

b) Altura total de las plantas (m)

En el segundo control, además de las variables mencionadas, se agregaron:

c) Diámetro de cuello, medido con pie de metro $(\mathrm{cm})$

d) Incremento en altura entre el primer y segundo año (m)

e) Un índice de crecimiento, como estimador de la biomasa, calculado a partir del diámetro de cuello al cuadrado multiplicado por la altura total ( $\mathrm{dc}^{2} \times \mathrm{H}$ ).

Análisis Estadístico. Debido a las restricciones impuestas por la heterogeneidad de los ensayos, en términos del tamaño de la unidad experimental, diseño estadístico y método de establecimiento, se éfectuó un análisis comparativo de las procedencias dentro de cada sitio 
exclusivamente. Para este propósito se seleccionaron las variables supervivencia e índice de crecimiento, de acuerdo con el control del segundo año.

Los valores promedio de las variables para cada procedencia fueron comparados estadísticamente a través de un Análisis de Varianza. En caso de existir diferencias significativas entre las procedencias, éstas se determinaron a través del Test de Comparación Múltiple de Tukey (Chun Li, 1969).

\section{CUADRO 3}

\section{ANTECEDENTES DEL ESTABLECIMIENTO DE LOS LUGARES DE ENSAYO Y DISEÑO EXPERIMENTAL}

\begin{tabular}{lcccc}
\hline & $\begin{array}{c}\text { Ensayo 1 } \\
\text { Llallauquén }\end{array}$ & $\begin{array}{c}\text { Ensayo 2 } \\
\text { Rinconada }\end{array}$ & $\begin{array}{c}\text { Ensayo 3 } \\
\text { Mel-Mel }\end{array}$ & $\begin{array}{c}\text { Ensayo 4 } \\
\text { Las Palmas }\end{array}$ \\
\hline Preparación del suelo & $\begin{array}{c}\text { Hoyo de } 30 \times \\
30 \times 30 \mathrm{~cm}\end{array}$ & $\begin{array}{c}\text { Hoyo de } 30 \times \\
30 \times 30 \mathrm{~cm}\end{array}$ & $\begin{array}{l}\text { Surcos en curvas } \\
\text { de nivel con trac- } \\
\text { ción animal. }\end{array}$ & $\begin{array}{c}\text { Hoyo de } 30 \mathrm{x} \\
30 \times 30 \mathrm{~cm}\end{array}$ \\
$\begin{array}{l}\text { Espaciamiento } \\
\begin{array}{l}\text { Unidad Experimental } \\
\text { (No de plantas) }\end{array}\end{array}$ & 20 & $3 \times 3 \mathrm{~m}$ & $2,5 \times 2,5 \mathrm{~m}$ & $2 \times 2 \mathrm{~m}$ \\
$\begin{array}{l}\text { Superficie (m }{ }^{2} \text { ) } \\
\text { Repeticiones }\end{array}$ & 88 & 20 & 16 & 20 \\
Diseño Estadístico & 3 & 198 & 156,25 & 88 \\
\hline
\end{tabular}

\section{RESULTADOS}

Los resultados obtenidos para cada uno de los ensayos se entregan en los Cuadros $\mathrm{N}^{\circ} 4,5$, 6 y 7 , ordenados según el Indice de Crecimiento. Para cada variable se entregan los valores mínimos, medios y máximos, además de su varianza.

\section{DISCUSION}

La experiencia desarrollada con procedencias de Eucalyptus camaldulensis reviste particular interés para la zona semiárida de Chile. Esta especie ha tenido buenos resultados de adaptación y crecimiento en zonas cuya pluviometría anual oscila entre 150 y $400 \mathrm{~mm}$. Introducida a fines del siglo pasado, probablemente junto con $E$. globulus, es común encontrarla en avenidas, cortinas cortavientos y pequeños bosquetes de la zona central chilena.

Los resultados del proyecto Introducción de Especies Forestales, desarrollado por INFOR a partir de 1962 , indican que $E$. camaldulensis presenta una buena adaptación en zonas edafoclimáticas con déficit hídrico. En la región mediterránea árida, con precipitaciones cercanas a los $150 \mathrm{~mm}$, ha destacado como una de las especies de mejor crecimiento, junto con $E$. Clodocalyx y $F$. sideroxylon.

En la región mediterránea semiárida, con una precipitación anual cercana a los $400 \mathrm{~mm}$, su crecimiento es similar al de $E$. globulus y sus subespecies $E$. globulus ssp bicostata y E. glo- 


\section{CUADRO 4 \\ RESULTADOS}

Procedencias Eucalyptus camaldulensis

Ensayo 1. Llallauquén

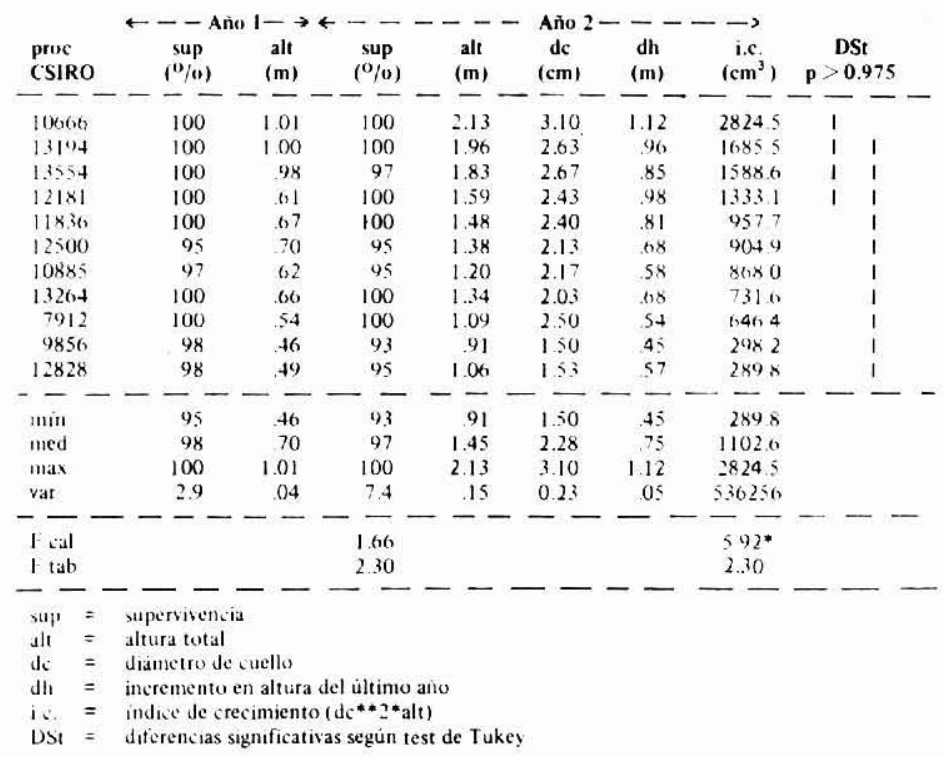

\section{CUADRO 5 \\ RESULTADOS}

Procedencias Eucalyptus camaldulensis Ensayo 2. Rinconada

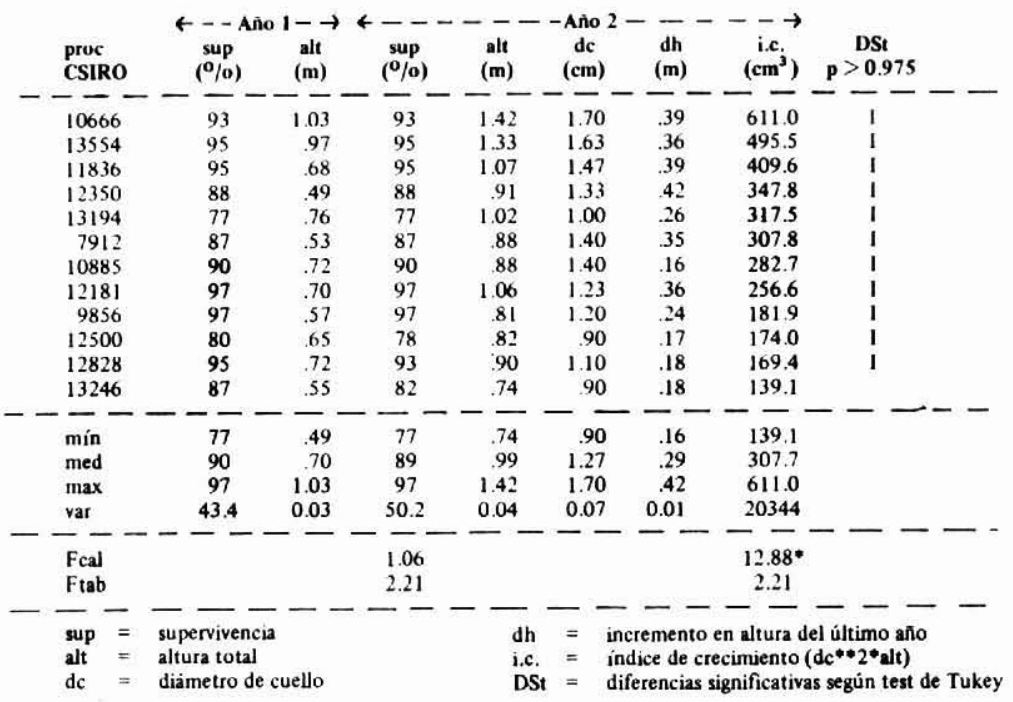




\section{CUADRO 6 \\ RESULTADOS \\ Procedencias Eucalyptus camaldulensis \\ Ensayo 3. Mel-Mel}

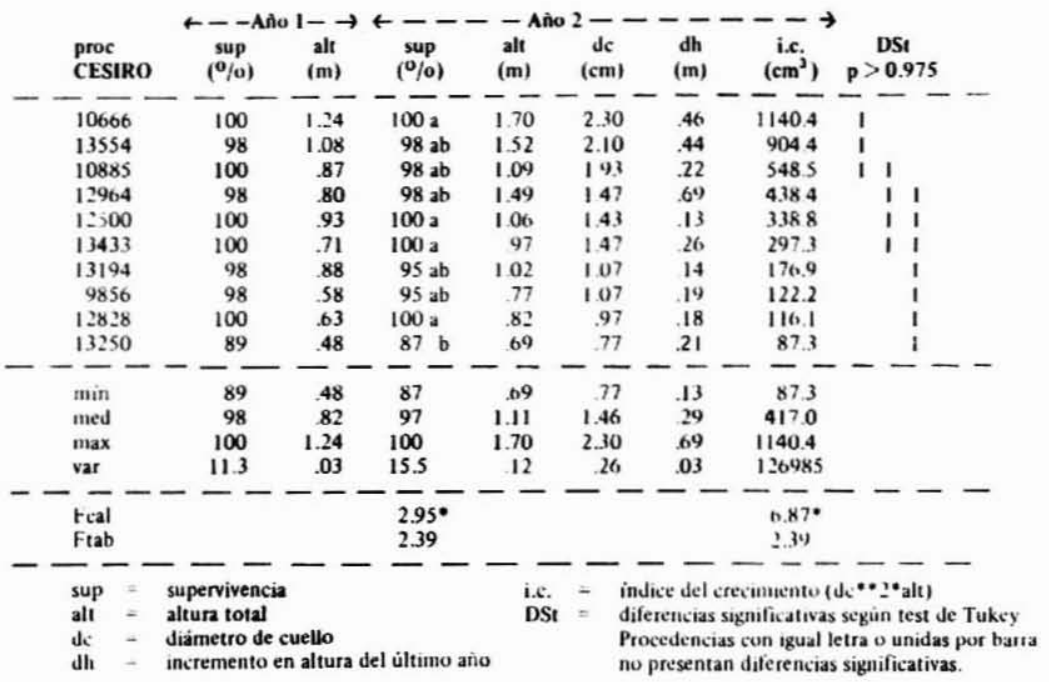

\section{CUADRO 7 \\ RESULTADOS \\ Procedencias Eucalyptus camaldulensis Ensayo 4. Las Palmas}

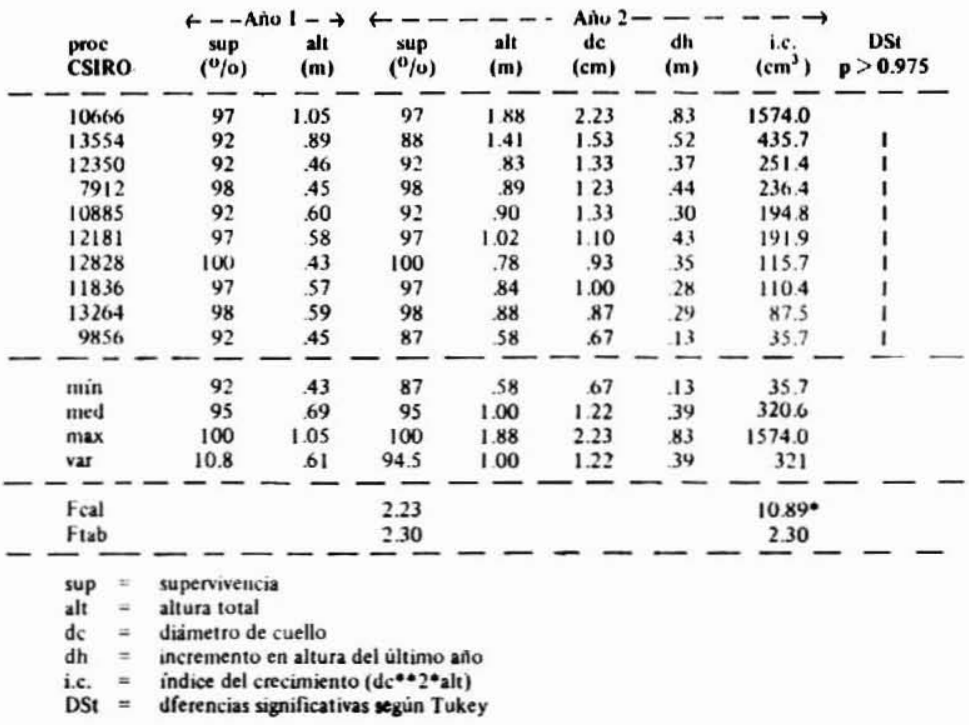


bulus ssp maidenii, aunque su forma es sólo de regular calidad.

Los resultados obtenidos permiten apreciar que la totalidad de las procedencias ensayadas en los 4 sitios muestran una buena supervivencia al cabo del segundo año, superior al $76 \%$ en todos los casos. Esto es de gran importancia debido a que la legislación de fomento forestal existente en el pais (D.L. NO 701 de 1974) otorga un subsidio del Estado a las plantaciones forestales a condición de que su supervivencia al primer año sea de $75 \%$ o más.

También es posible observar que existe una alta heterogeneidad en términos de tolerancia al medio (resistencia a la sequía, heladas, ataques de insectos), vigor, forma y tasas de crecimiento entre las procedencias, ya que se registran diferencias notables en términos de incremento en algura, diámetro y volumen (Figura $\mathrm{N}^{\mathrm{O}} 2$ ).

\section{FIGURA 2 \\ INDICE DE CRECIMIENTO DE LAS PROCEDENCIAS DE Eucalyptus camaldulensis}

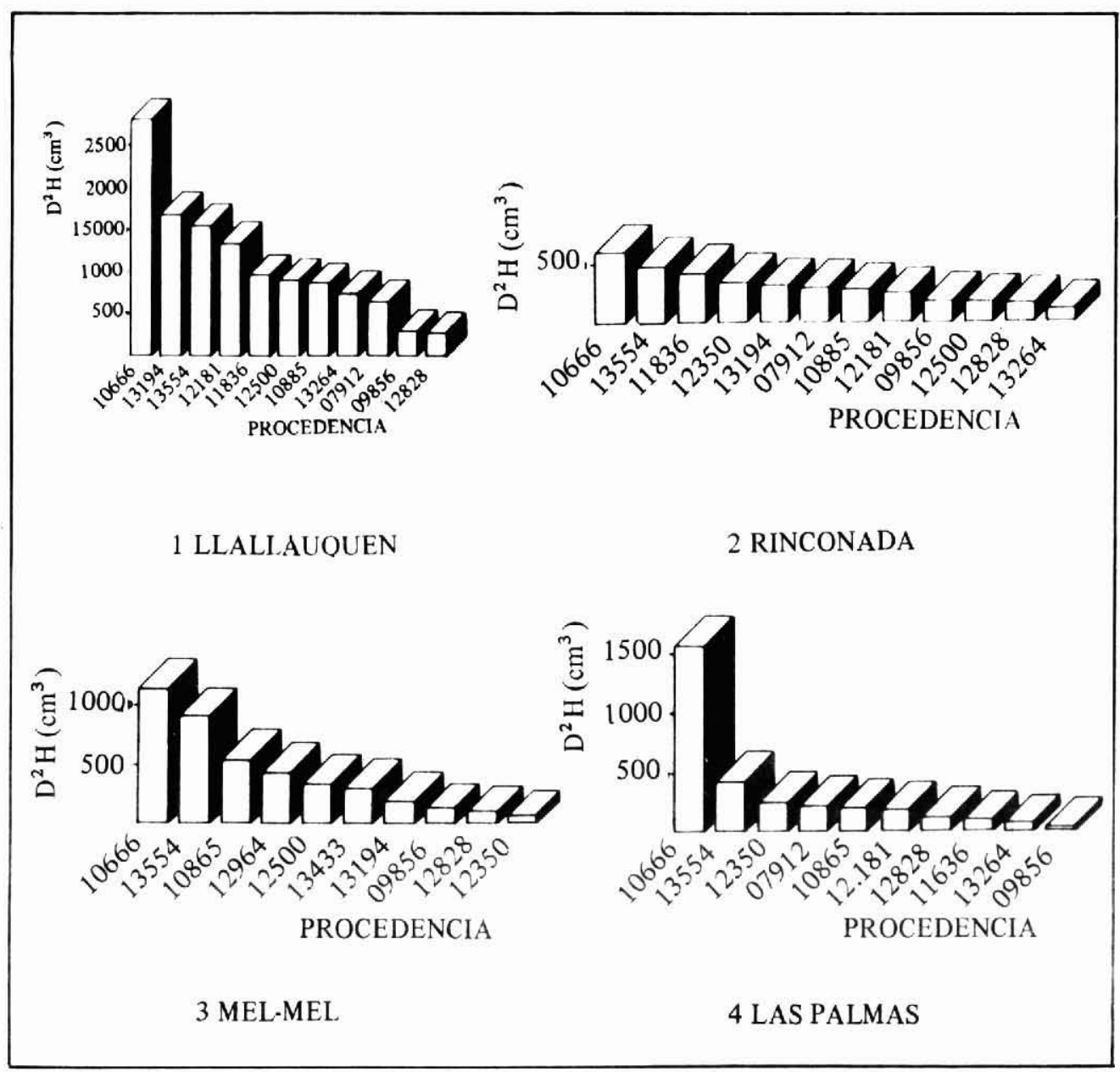


La procedencia de mejor resultado para la zona de estudio, por su plasticidad ecológica y su productividad, es Lake Albacutya, Victoria (Código 10666 CSIRO). En todos los ensayos mostró los mayores crecimientos en altura y en volumen. En el lugar con mejor resultado (Llallauquén), su altura promedio a los 2 años era de $2,13 \mathrm{~m}$ con un incremento anual de 1,12 $\mathrm{m}$. Su forma es bastante aceptable para la producción de postes y no se evidencian problemas fitosanitarios (Fotos 1,2 y 3 ).

Las procedencias de menor crecimiento en todos los ensayos fueron las de Agnew, Western Australia (Código 9856); NW Dodnadatta, Southern Australia (Código 12828); Nof Quilpio, Queensland (Código 12828); Nof Quilpio, Queensland (Código 13264) y Fitzroy River Crossiana, Western Australia (Código 13250).

Aunque el plazo de experimentación es reducido, las significativas diferencias que existen entre los resultados obtenidos con la procedencia 10666, Lake Albacutya, y el resto, en todos los sitios ensayados, permiten recomendar que las próximas experiencias se refieran a ensayos silvícolas efectuando pequeñas plantaciones de la procedencia seleccionada, dejando de lado la fase de Alcance Restringido, sugerida por Burley y Wood (1979).

Los futuros ensayos debieran orientarse al ensayo de técnicas de establecimiento más intensivas para la repoblación con Eucalyptos camaldulensis y tratamientos silviculturales en función de diferentes utilizaciones de su madera.

El empleo de arados o subsoladores, la fertilización y un buen control de la competencia incidirían en un mejor resultado de las plantaciones de $E$. camaldulensis, como ha sido demostrado en experiencias con E. globulus (Prado y Rojas, 1985) en la Zona Semiárida de Chile.

\section{CONCLUSIONES}

La procedencia de mayor plasticidad ecológica, en términos de supervivencia, crecimiento, forma y productividad, fue Lake Albacutya, Victoria (10666).

- La totalidad de las procedencias ensayadas alcanzaron una supervivencia superior al $76 \%$ en todos los sitios.

Las diversas procedencias ensayadas presentan una alta heterogeneidad en términos de su tolerancia al medio, vigor, forma y tasa de crecimiento.

Las procedencias de $E$. camaldulensis de menor crecimiento entre las ensayadas fueron la de Agnew, Western Australia (9856); Dodnadatta, Southern Australia (12828); Quilpio, Queensland (13264) y Fitzroy River Crossiana, Western Australia (13250). 


\section{REFERENCIAS BIBLIOGRAFICAS}

BURLEY, J, and WOOD, P.J., comp. 1976. A manual on species and provenance research with particular reference to the Tropics. Commonwealth Forestry Institute, Univ. Oxford. Tropical Forestry Papers No 10.226 p.

L, CH.CH. 1969. Introducción a la estadística experimental. Barcelona, Ed. Omega, 496 p.

DI CASTRI, F. 1975. Esbozo ecológico de Chile. Santiago, Chile, Centro de Perfeccionamiento, Experimentación e Investigaciones Pedagógicas. 64 p.

ELDRIDGE, K.G., comp. An annotated bibliography of genetic variation in Eucalyptus camaldulensis. Commonwealth Forestry Institute, Univ. Oxford and CSIRO, Australia, Tropical Forestry Papers No $8.59 \mathrm{p}$.

FAO. 1979. Eucalyptus for planting. FAO, Rome. FAO Forestry Series $N^{0} 11.677 \mathrm{p}$.

GHOSH, R.C.; GUPTA, B.N. and SHEDHA, M.D. 1977. Provenance trial of Eucalyptus camaldulensis Dehn. The Indian For 103(7):442-453.

GOOR, A.Y. and BARNEY, C.W. 1976. Forest tree planting in arid zones, 2a. ed. New York, The Ronadl Press Co. 504 p.

INSTITUTO FORESTAL y UNIVERSIDAD DE CHILE, FAC. CS. FOR, 1979. Areas cubiertas por ensayos de introducción de especies y ubicación de nuevas experiencias. Informe II. Proyecto CONAF/ PNUD/FAO/CHI-76-003, Santiago, Chile. 87 p., apéndices.

INSTITUTO FORESTAL/CORPORACION DE FOMENTO DE LA PRODUCCION. Especies forestales exóticas de interés económico para Chile. INFOR/CORFO, Santiago, Chile. 168 p.

DARROW, W.K. 1983. Provenance- type trials of Eucalyptus camaldulensis and E. tereticomis in South Africa and South-West Africa: Eight-year results. S. Afr. For, J. No 124:13-22.

NATIONAL ACADEMY OF SCIENCES. 1980. Firewood crops. Shrub and tree species for energy production. Washington, Nat. Ac. of Sci. 237 p.

NAWAZ, M. 1963. Introduction of fast growing tree species in West Pakistan. Lahore, Government of West Pakistan. $74 \mathrm{p}$.

PRADO, J,A. y ROJAS, P. 1985. Efecto de la preparación del suelo, fertilización y control de la competencia en el establecimiento de plantaciones de Eucalyptus globulus en la Zona Semiárida de Chile. En: Actas Segundo Encuentro Regional C.I.I.D. Forestación en zonas áridas y semiáridas. Santiago, Chile. pp. 234-247.

ROBERTS, R.C. y DIAZ V., C. 1960. Los grandes grupos de suelos de Chile. Agr. Tec. (Santiago, Chile). v. 19 y $20: 7-36$.

SIDDIQUI, K.M.; KHAN, M. and AKHTAR, S. 1979. Results of 10-year old Eucalyptus camaldulensis Dehn. provenance study at Peshawar. Silvae Genet, 28(1):24-26.

TURNBULL, J.W. and PRYOR, L.D. 1978. Choice of species and seed sources, In: Hillis, W.E. and Brown, A.G., ed. Eucalyp tus for wood production. Australia, CSIRO. pp. 6-65. 